\title{
A Study of Some Geometric Properties of Meromorphic Univalent Functions Associated with Ruscheweyh Derivative
}

\author{
Kassim A. Jassim ${ }^{1}$, Faeza Khaleel Shabeeb ${ }^{2}$ \\ ${ }^{1,2}$ College of Science, Department of Mathematics-University of Baghdad
}

\begin{abstract}
We presented in this paper a new class $S(\lambda, \alpha, \beta)$ containing meromorphic univalent in functions in the punctured unit disk. We obtained many geometric properties, likes coefficient inequality, distortion and growth theorems, radii of starlikeness and convexity, extreme points, convex linear combination, arithmetic mean, hadamard product, closure theorems, neighborhood and partial sums results.
\end{abstract}

Keywords: Univalent functions, meromorphic functions, starlike and convex functions.

\section{Introduction}

Let $\mathrm{S}$ denote the class of functions of the from

$$
f(\mathrm{z})=\mathrm{z}^{-1}+\sum_{n=1}^{\infty} a_{n} z^{n}, a_{n} \geq 0, n \in N=\{1,2, \ldots\}
$$

which are meromorphic univalent functions in the punctured unit disk $U^{*}=\{\mathrm{z} \in \mathbb{C} ; 0<|\mathrm{z}|<1\}$. The hadamard product (or convolution) of two functions $f$ and $g$

$$
\left(f^{*} g\right)(\mathrm{z})=\mathrm{z}^{-1}+\sum_{n=1}^{\infty} a_{n} b_{n} z^{n},
$$

where $g(\mathrm{z})=\mathrm{z}^{-1}+\sum_{n=1}^{\infty} b_{n} z^{n}, \quad b_{n} \geq 0$

Also, a function $f \in S$ is meromorphic starlike function of order $\delta(0 \leq \delta<1)$ if

$-R e\left\{\frac{Z f^{\prime}(Z)}{f(z)}\right\}>\delta$, for $z \in U^{*}$,

and a function $f \in S$ is meromorphic convex of order $\delta(0$ $\leq \delta<1)$ if

$$
-\operatorname{Re}\left\{1+\frac{Z f^{\prime \prime}(Z)}{f^{\prime}(z)}\right\}>\delta, \text { for } z \in U^{*} .
$$

The Ruscheweyh derivative of order $\lambda$ is denoted by $D^{\lambda} f$ and is defined as follows

$$
\begin{aligned}
& D^{\lambda} f(z)=\frac{1}{z(1-z)^{\lambda+1}} * f(z)= \\
& z+\sum_{n=p}^{\infty} D_{n}(\lambda) a_{n} z^{n}, \lambda>-1, z \in U^{*} .
\end{aligned}
$$

where

$$
D_{n}(\lambda)=\frac{(\lambda+1)(\lambda+2) \ldots(\lambda+n+1)}{(n+1) !}
$$

Our aim of thispaper is to study the class $S(\lambda, \alpha, \beta)$ containing of function $f$ and satisfying

$$
\left|\frac{\frac{z\left(D^{\lambda} f(z)\right)^{\prime}}{D^{\lambda} f(z)}+1}{\frac{2 z\left(D^{\lambda} f(z)\right)^{\prime}}{D^{\lambda} f(z)}+2 \alpha}\right|<\beta
$$

Where

$$
D^{\lambda} f(z)=z^{-1}+\sum_{n=1}^{\infty} D_{n}(\lambda) a_{n} z^{n} .
$$

Many authors studied classes of meromorphic functions which are univalent and multivalent like W. G. Atshan in $[1,2]$, B. A. Frasin and M. Darus in [3],A. R. S. Juma and H.
Zirar in [4] ,L. Liu in [5], L. Liu and M. Srivastava in [6], J. E. Miller in [7] , and M. L. Mogra in [8],R. K. Raina and H. M. Srivastava in [9] and N. Xu and D. Yang in [10].

\section{Coefficient Inequality}

Theorem (2.1) Let the function $f$ defined by (1.1). Then $f \in \mathrm{S}(\lambda, \alpha, \beta)$ if and only if

$\sum_{n=1}^{\infty}[n+1+2 \beta(n+\alpha)] D_{n}(\lambda) a_{n}<2 \beta(1-\alpha)(2.1)$ where , $0 \leq \alpha<1,0<\beta \leq 1$.

Proof. Assume the condition (2.1) is satisfied, therefore $\left|z\left(D^{\lambda} f(z)\right)^{\prime}+\left(D^{\lambda} f(z)\right)\right|$

$$
\begin{gathered}
-\beta\left|2 z\left(D^{\lambda} f(z)\right)^{\prime}+2 \alpha\left(D^{\lambda} f(z)\right)\right| \\
=\left|-z^{-1}+\sum_{n=1}^{\infty} D_{n}(\lambda) n a_{n} z^{n}+z^{-1}+\sum_{n=1}^{\infty} D_{n}(\lambda) a_{n} z^{n}\right|- \\
\beta \mid-2 z^{-1}+2 \sum_{n=1}^{\infty} D_{n}(\lambda) n a_{n} z^{n}+2 \alpha z^{-1} \\
+2 \alpha \sum_{n=1}^{\infty} D_{n}(\lambda) a_{n} z^{n} \mid \\
=\left|\sum_{n=1}^{\infty} D_{n}(\lambda)(n+1) a_{n} z^{n}\right|-\beta \mid 2(1-\alpha) z^{-1}- \\
2 n=1 \infty D n(\lambda)(n+\alpha) a n z n \\
\leq \sum_{n=1}^{\infty} D_{n}(\lambda)(n+1) a_{n}+2 \beta \sum_{n=1}^{\infty} D_{n}(\lambda)(n+\alpha) a_{n} \\
\quad-2 \beta(1-\alpha) \\
\leq \sum_{n=1}^{\infty} D_{n}(\lambda)[n+1+2 \beta(n+\alpha)] a_{n}-2 \beta(1-\alpha) \leq 0 .
\end{gathered}
$$

Then by maximum modulus theorem, we get the result.

For the Converse, assume that $\left|\frac{\frac{z\left(D^{\lambda} f(z)\right)^{\prime}}{D^{\lambda} f(z)}+1}{\frac{2 z\left(D^{\lambda} f(z)\right)^{\prime}}{D^{\lambda} f(z)}+2 \alpha}\right|<\beta$ is satisfied. Therefore,

$\left|\frac{\sum_{n=1}^{\infty} D_{n}(\lambda)(n+1) a_{n} z^{n}}{2(1-\alpha) z^{-1}-2 \sum_{n=1}^{\infty} D_{n}(\lambda)(n+\alpha) a_{n} z^{n}}\right|<\beta$. Since $|\operatorname{Re}(z)| \leq|z|$ for all $\mathrm{z}$, we have

$\operatorname{Re}\left\{\frac{\sum_{n=1}^{\infty} D_{n}(\lambda)(n+1) a_{n} z^{n}}{2(1-\alpha) z^{-1}-2 \sum_{n=1}^{\infty} D_{n}(\lambda)(n+\alpha) a_{n} z^{n}}\right\}<\beta$. Then by choosing the value of $\mathrm{z}$ on the real axis and letting $\mathrm{z} \rightarrow 1-$ through values , we get

$$
\sum_{n=1}^{\infty}[n+1+2 \beta(n+\alpha)] D_{n}(\lambda) a_{n}<2 \beta(1-\alpha) .
$$




\section{International Journal of Science and Research (IJSR) \\ ISSN (Online): 2319-7064}

Index Copernicus Value (2015): 78.96 | Impact Factor (2015): 6.391

Corollary (2.1) If $f \in \mathrm{S}(\lambda, \alpha, \beta)$, then

$a_{n} \leq \frac{2 \beta(1-\alpha)}{[n+1+2 \beta(n+\alpha)] D_{n}(\lambda)}(2.2)$

where, $0 \leq \alpha<1,0<\beta \leq 1$.

\section{Distortion and Growth Theorem}

Theorem (3.1) If the function $f$ defined by (1.1) is in the class $S(\lambda, \alpha, \beta)$, then for $0<|z|=r<1$, we have

$$
|f(z)| \leq \frac{1}{r}+\frac{\beta(1-\alpha)}{1+\beta(1+\alpha)} r \text { and }|f(z)| \geq \frac{1}{r}-\frac{\beta(1-\alpha)}{1+\beta(1+\alpha)} r
$$

\section{Proof}

Since $f(z)$ be a function in $S(\lambda, \alpha, \beta)$, then we have from Theorem $(2.1)$

Then

$$
\sum_{n=1}^{\infty}[n+1+2 \beta(n+\alpha)] D_{n}(\lambda) a_{n}<2 \beta(1-\alpha) .
$$

$$
|f(z)|=\left|z^{-1}+\sum_{n=1}^{\infty} a_{n} z^{n}\right| \leq\left|z^{-1}\right|+\sum_{n=1}^{\infty} a_{n}|z|^{n}
$$

$\leq \frac{1}{r}+r \sum_{n=1}^{\infty} a_{n}$,

By theorem (2.1),

we get $\sum_{n=1}^{\infty}\left|a_{n}\right| \leq \frac{2 \beta(1-\alpha)}{[1+\beta(1+\alpha)](\lambda+1)(\lambda+2)}$.

Then

Also,

$$
|f(z)| \leq \frac{1}{r}+\frac{2 \beta(1-\alpha)}{[1+\beta(1+\alpha)](\lambda+1)(\lambda+2)} r
$$

$$
|f(z)| \geq \frac{1}{r}-\frac{2 \beta(1-\alpha)}{[1+\beta(1+\alpha)](\lambda+1)(\lambda+2)} r .
$$

Corollary (3.2) If the function $f$ defined by (1.1) is in the class $S(\lambda, \alpha, \beta)$, then for $0<|z|=r<1$, we have $\frac{1}{r^{2}}-\frac{2 \beta(1-\alpha)}{[1+\beta(1+\alpha)](\lambda+1)(\lambda+2)} \leq\left|f^{\prime}(z)\right| \leq \frac{1}{r^{2}}+\frac{2 \beta(1-\alpha)}{[1+\beta(1+\alpha)](\lambda+1)(\lambda+2)}$

\section{Radii of Star Likeness and Convexity}

The following results giving the radii of starlikeness and convexity of the function $f(z) \in \mathrm{S}(\lambda, \alpha, \beta)$.
Theorem (4.1) If a function $f \in \mathrm{S}(\lambda, \alpha, \beta)$, then $f$ is meromorphically starlike function of order $\delta, 0 \leq \delta<1$ in the disk $|z|<R_{1}$, where

$$
R_{1}=\inf _{n}\left[\frac{[n+1+2 \beta(n+\alpha)] D_{n}(\lambda)(1-\delta)}{2 \beta(1-\alpha)(n+\delta+2)}\right]^{\frac{1}{n+1}}
$$

Proof

It is sufficient to show that

$$
\begin{aligned}
& \left|\frac{z f^{\prime}(Z)}{f(Z)}+1\right|=\left|\frac{Z f^{\prime}(z)}{f(z)}+1\right| \leq 1-\delta, \\
& =\left|\frac{-Z^{-1}+\sum_{n=1}^{\infty} n a_{n} z^{n}+Z^{-1}+\sum_{n=1}^{\infty} a_{n} z^{n}}{Z^{-1}+\sum_{n=1}^{\infty} a n z^{n}}\right| \\
& =\left|\frac{\sum_{n=1}^{\infty}(n+1) a_{n} z^{n+1}}{1+\sum_{n=1}^{\infty} a_{n} z^{n+1}}\right| \leq \frac{\sum_{n=1}^{\infty}(n+1) a_{n}|z|^{n+1}}{1-\sum_{n=1}^{\infty} a_{n}|z|^{n+1}}
\end{aligned}
$$

The last expression must bounded by $1-\delta$ if

$$
\begin{gathered}
\sum_{n=1}^{\infty}(n+1) a_{n}|z|^{n+1} \leq 1-\delta-(1-\delta) \sum_{n=1}^{\infty} a_{n}|z|^{n+1}, \\
\sum_{n=1}^{\infty}(n+\delta+2) a_{n}|z|^{n+1} \leq(1-\delta) .
\end{gathered}
$$

Then by theorem(2.1), we have

Hence

$$
\sum_{n=1}^{\infty} \frac{[n+1+2 \beta(n+\alpha)] D_{n}(\lambda)}{2 \beta(1-\alpha)} a_{n}<1 .
$$

Also,

$$
|z|^{n+1} \leq \frac{[n+1+2 \beta(n+\alpha)] D_{n}(\lambda)(1-\delta)}{2 \beta(1-\alpha)(n+\delta+2)},
$$

Therefore, we get the result

$$
|z| \leq\left[\frac{[n+1+2 \beta(n+\alpha)] D_{n}(\lambda)(1-\delta)}{2 \beta(1-\alpha)(n+\delta+2)}\right]^{\frac{1}{n+1}} .
$$

Theorem (4.2). If a function $f \in \mathbf{S}(\lambda, \alpha, \beta)$, then $f$ is meromorphically convex function of order $\delta,(0 \leq \delta<1)$ in the disk $|z|<R_{2}$, where

$$
R_{2}=\underset{n}{\operatorname{in} f}\left[\frac{[n+1+2 \beta(n+\alpha)](1-s)}{2 n \beta(1-\alpha)(n+\delta+2)}\right]^{n+1}
$$

Proof. It is sufficient to show that

$$
\begin{aligned}
\left|\frac{z f^{\prime \prime}(z)+2 f^{\prime}(z)}{f^{\prime}(z)}\right|= & \left|\frac{2 Z^{-2}+\sum_{n=1}^{\infty} n(n-1) a_{n} Z^{n-1}-2 Z^{-2}+2 \sum_{n=1}^{\infty} n a_{n} Z^{n-1}}{-Z^{-2}+\sum_{n=1}^{\infty} n a_{n} z^{n-1}}\right| \\
& =\left|\frac{\sum_{n=1}^{\infty} n(n+1) a_{n} z^{n+1}}{-1+\sum_{n=1}^{\infty} n a_{n} z^{n+1}}\right| \leq \frac{\sum_{n=1}^{\infty} n(n+1) a_{n}|z|^{n+1}}{1-\sum_{n=1}^{\infty} n a_{n}|z|^{n+1}} .
\end{aligned}
$$

The last expression must bounded by $1-\delta$.Therefore,

$$
\begin{gathered}
\sum_{n=1}^{\infty} n(n+1) a_{n}|z|^{n+1} \leq 1-\delta \\
-(1-\delta) \sum_{n=1}^{\infty} n a_{n}|z|^{n+1} \\
\sum_{n=1}^{\infty} n(n+\delta+2) a_{n}|z|^{n+1} \leq 1-\delta
\end{gathered}
$$

Then by theorem $(2.1)$, we have

Hence
Also,

$$
|z|^{n+1} \leq \frac{[n+1+2 \beta(n+\alpha)] D_{n}(\lambda)(1-\delta)}{2 \beta(1-\alpha) n(n+\delta+2)},
$$

$$
|\mathrm{z}| \leq\left[\frac{[n+1+2 \beta(n+\alpha)] D_{n}(\lambda)(1-s)}{2 \beta(1-\alpha) n(n+\delta+2)}\right]^{n+1}
$$

Therefore, we get the result.

\section{Convex Linear Combination}

In the following theorem, the class $S(\lambda, \alpha, \beta)$ is closed under convex linear combination.

\section{Volume 6 Issue 7, July 2017 www.ijsr.net}




\section{International Journal of Science and Research (IJSR) \\ ISSN (Online): 2319-7064 \\ Index Copernicus Value (2015): 78.96 | Impact Factor (2015): 6.391}

Theorem (5.1) . The class $S(\lambda, \alpha, \beta)$ is closed under convex linear combination.

Proof: We want to show that function $\mathrm{k}(\mathrm{z})=(1-$ ? $) f_{1}(\mathrm{z})+$ ? $f_{2}(\mathrm{z}), 0 \leq$ ? $\leq 1$ is in the class $\mathrm{S}(\lambda, \alpha, \beta)$ where $f_{1}(\mathrm{z})$, $f_{2}(\mathrm{z}) \in \mathrm{S}(\lambda, \alpha, \beta)$ and

$$
f_{1}(\mathrm{z})=z^{-1}+\sum_{n=1}^{\infty} a_{n} z^{n}, f_{2}(\mathrm{z})=z^{-1}+\sum_{n=1}^{\infty} b_{n} z^{n},
$$

where $a_{n}, b_{n} \geq 0$.

By theorem (2.1) we have

$$
\sum_{n=1}^{\infty}[n+1+2 \beta(n+\alpha)] D_{n}(\lambda) a_{n} \leq 2 \beta(1-\alpha)
$$

and

$$
\sum_{n=1}^{\infty}[n+1+2 \beta(n+\alpha)] D_{n}(\lambda) b_{n} \leq 2 \beta(1-\alpha) .
$$

Therefore,

$\mathrm{k}(\mathrm{z})=(1-$ ? $) f_{1}(\mathrm{z})+$ ? $f_{2}(\mathrm{z})$

$=(1-$ ? $)\left(z^{-1}+\sum_{n=1}^{\infty} a_{n} z^{n}\right)+$ ? $\left(z^{-1}+\sum_{n=1}^{\infty} b_{n} z^{n}\right)$

$=z^{-1}+\sum_{n=1}^{\infty}\left[(1-\right.$ ? $) a_{n}+$ ? $\left.b_{n}\right] z^{n}$. Further

$\sum_{n=1}^{\infty}[(n+1)+2 \beta(n+\alpha)]\left[(1-\right.$ ? $) a_{n}+$ ? $\left.b_{n}\right] D_{n}(\lambda)$

$=\sum_{n=1}^{\infty}[n+1+2 \beta(n+\alpha)](1-$ ? $) a_{n}+\sum_{n=1}^{\infty}[n+1+$

$2 \beta(n+\alpha)$ ? bn

$\leq 2(1-\mu) \beta(1-\alpha)+2 \mu \beta(1-\alpha)=2 \beta(1-\alpha)$,

Therefore the result is follows that $\mathrm{k}(\mathrm{z})$ is in the class $\mathrm{S}(\lambda, \alpha, \beta)$

\section{Arithmetic Mean}

In the following, we shall prove that class $S(\lambda, \alpha, \beta)$ is closed under arithmetic mean.

Theorem (6.1).Let $f_{1}(\mathrm{z}), f_{2}(\mathrm{z}) \ldots \ldots f_{l}(\mathrm{z})$ defined by $f_{i}(\mathrm{z})=$ $z^{-1}+\sum_{n=1}^{\infty} a_{n, i} z^{n}, \quad\left(a_{n, i} \geq 0, i=1,2 \ldots \ldots l\right)$ be in the class $\mathrm{S}(\lambda, \alpha, \beta)$. Then the Arithmetic mean of $f_{i}(\mathrm{z}),(\mathrm{i}=1 \ldots . l)$ defined by

$$
h(\mathrm{z})=\frac{1}{l} \sum_{i=1}^{l} f_{i}(\mathrm{z})
$$

is also in the class $S(\lambda, \alpha, \beta)$.

\section{Proof}

By hypothesis we get

$h \quad(\mathrm{z})=\frac{1}{l} \sum_{i=1}^{l}\left(z^{-1}+\sum_{n=1}^{\infty} a_{n, i} z^{n}\right)=z^{-1}+$ $\sum_{n=1}^{\infty}\left(\frac{1}{l} \sum_{i=1}^{l} a_{n, i}\right) z^{n}$

since $f_{i}(\mathrm{z}) \in \mathrm{S}(\lambda, \alpha, \beta)$ for every $i=1,2, \ldots$ l. So by using theorem (2.1), we get

that $\sum_{n=1}^{\infty}[n+1+2 \beta(n+\alpha)] D_{n}(\lambda)\left(\frac{1}{l} \sum_{i=1}^{l} a_{n, i}\right)$

$=\frac{1}{l} \sum_{i=1}^{l}\left(\sum_{n=1}^{\infty}[n+1+2 \beta(n+\alpha)] D_{n}(\lambda) a_{n, i}\right)$

$\leq \frac{1}{l} \sum_{i=1}^{l} 2 \beta(1-\alpha)=2 \beta(1-\alpha)$

\section{Hadamard Product}

In the following theorem, we obtain the convolution result for function belongs to the class $S(\lambda, \alpha, \beta)$.
Theorem (7.1). Let the functions $f, g$ of the form (1.1) be in the class $\mathrm{S}(\lambda, \alpha, \beta)$. Then $(f * g) \in \mathrm{S}(\lambda, \alpha, \beta)$.Then $(f * g) \in$ $\mathrm{S}(\lambda, \alpha, l)$, where

$$
l \geq \frac{2 \beta^{2}(1-\alpha)(n+1)}{[n+1+2 \beta(n+\alpha)]^{2} D_{n}(\lambda)-4 \beta^{2}(1-\alpha)(n+\alpha)}
$$

Proof

Let $f, g \in \mathrm{S}(\lambda, \alpha, \beta)$ and so

and

$$
\sum_{n=1}^{\infty} \frac{[n+1+2 \beta(n+\alpha)] D_{n}(\lambda)}{2 \beta(1-\alpha)} a_{n} \leq 1
$$

$$
\sum_{n=1}^{\infty} \frac{[n+1+2 \beta(n+\alpha)] D_{n}(\lambda)}{2 \beta(1-\alpha)} b_{n} \leq 1
$$

We have to find the smallest number $l$ such that

$$
\sum_{n=1}^{\infty} \frac{[n+1+2 l(n+\alpha)] D_{n}(\lambda)}{2 l(1-\alpha)} a_{n} b_{n} \leq 1
$$

By the Cauchy-Schwartz inequality, we get

$$
\sum_{n=1}^{\infty} \frac{[n+1+2 \beta(n+\alpha)] D_{n}(\lambda)}{2 \beta(1-\alpha)} \sqrt{a_{n} b_{n}} \leq 1(7.4)
$$

It is sufficient to show that

$$
\frac{[n+1+2 l(n+\alpha)] D_{n}(\lambda)}{2 l(1-\alpha)} a_{n} b_{n} \leq \frac{[n+1+2 \beta(n+\alpha)] D_{n}(\lambda)}{2 \beta(1-\alpha)} \sqrt{a_{n} b_{n}}
$$

That is,

$$
\sqrt{a_{n} b_{n}} \leq \frac{l[n+1+2 \beta(n+\alpha)]}{\beta[n+1+2 l(n+\alpha)]}
$$

But from (7.4)

$$
\sqrt{a_{n} b_{n}} \leq \frac{2 \beta(1-\alpha)}{[n+1+2 \beta(n+\alpha)] D_{n}(\lambda)}
$$

Thus it is enough to show that

$$
\frac{2 \beta(1-\alpha)}{[n+1+2 \beta(n+\alpha)] D_{n}(\lambda)} \leq \frac{l[n+1+2 \beta(n+\alpha)]}{\beta[n+1+2 l(n+\alpha)]}
$$

which simplifies to

$$
l \geq \frac{2 \beta^{2}(1-\alpha)(n+1)}{[n+1+2 \beta(n+\alpha)]^{2} D_{n}(\lambda)-4 \beta^{2}(1-\alpha)(n+\alpha)}
$$

which proves theorem(7.1)

Theorem (7.2).Let the functions $f, g$ of the form (1.1) be in the class $\mathrm{S}(\lambda, \alpha, \beta)$. Then the function $h(z)=z^{-1}+$ $\sum_{n=1}^{\infty}\left(a_{n}^{2}+b_{n}^{2}\right) z^{n}$ is in the class $\mathrm{S}(\lambda, \alpha, \gamma)$, where

$$
\gamma=\frac{4 \beta^{2}(n+1)(1-\alpha)^{2}}{(1-\alpha)[n+1+2 \beta(n+\alpha)]^{2} D_{n}(\lambda)-8 \beta^{2}(n+\alpha)(1-\alpha)^{2}}
$$

Proof. Since $f, g \in \mathrm{S}(\lambda, \alpha, \beta)$, therefore , by theorem (2.1) yields

and

$$
\sum_{n=1}^{\infty}\left[\frac{[n+1+2 \beta(n+\alpha)] D_{n}(\lambda)}{2 \beta(1-\alpha)}\right]^{2} a_{n}^{2} \leq 1
$$

$$
\sum_{n=1}^{\infty}\left[\frac{[n+1+2 \beta(n+\alpha)] D_{n}(\lambda)}{2 \beta(1-\alpha)}\right]^{2} b_{n}^{2} \leq 1
$$

We obtain from the last two inequalities

$$
\sum_{n=1}^{\infty} \frac{1}{2}\left[\frac{[n+1+2 \beta(n+\alpha)] D_{n}(\lambda)}{2 \beta(1-\alpha)}\right]^{2}\left(a_{n}^{2}+b_{n}^{2}\right) \leq 1
$$

But $h(z) \in \mathrm{S}(\lambda, \alpha, \gamma)$ if and only if

\section{Volume 6 Issue 7, July 2017 www.ijsr.net}




\section{International Journal of Science and Research (IJSR) \\ ISSN (Online): 2319-7064}

Index Copernicus Value (2015): 78.96 | Impact Factor (2015): 6.391

$$
\sum_{n=1}^{\infty} \frac{[n+1+2 \gamma(n+\alpha)] D_{n}(\lambda)}{2 \gamma(1-\alpha)}\left(a_{n}^{2}+b_{n}^{2}\right)
$$

Therefore, the inequality (7.9) satisfied if

$$
\begin{aligned}
& \frac{[n+1+2 \gamma(n+\alpha)] D_{n}(\lambda)}{2 \gamma(1-\alpha)} \\
& \leq \frac{1}{2}\left[\frac{[n+1+2 \beta(n+\alpha)] D_{n}(\lambda)}{2 \beta(1-\alpha)}\right]^{2}
\end{aligned}
$$

which is imply

$\gamma \geq \frac{4 \beta^{2}(n+1)(1-\alpha)^{2}}{(1-\alpha)[n+1+2 \beta(n+\alpha)]^{2} D_{n}(\lambda)-8 \beta^{2}(n+\alpha)(1-\alpha)^{2}}$ which is complete the proof.

\section{Closure Theorem}

We shall prove the following closure theorems for the class $\mathrm{S}(\lambda, \alpha, \beta)$.

Theorem (8.1). Let $f_{j} \in \mathrm{S}(\lambda, \alpha, \beta), \mathrm{j}=1,2 \ldots s$, then

$$
g(z)=\sum_{j=1}^{s} c_{j} f_{j}(z) \in \mathrm{S}(\lambda, \alpha, \beta),
$$

for $f_{j}(z)=z^{-1}+\sum_{n=1}^{\infty} a_{n, j} z^{n}$, where $\sum_{j=1}^{s} c_{j}=1$.

Proof. Suppose that $g(z)=\sum_{j=1}^{s} c_{j} f_{j}(z)$. Since $f_{j}(z)=$ $z^{-1}+\sum_{n=1}^{\infty} a_{n, j} z^{n}$,

Therefore,

$\sum_{j=1}^{S} c_{j} a_{n, j}$.

$$
g(z)=z^{-1}+\sum_{n=1}^{\infty} e_{n} z^{n}, \text { where } e_{n}=
$$

Thus

$g(z) \in \mathrm{S}(\lambda, \alpha, \beta)$ if $\sum_{n=1}^{\infty} \frac{[n+1+2 \beta(n+\alpha)] D_{n}(\lambda)}{2 \beta(1-\alpha)} e_{n} \leq 1$.That is, if

$$
\begin{aligned}
& \sum_{n=1}^{\infty} \sum_{j=1}^{s} \frac{[n+1+2 \beta(n+\alpha)] D_{n}(\lambda)}{2 \beta(1-\alpha)} c_{j} a_{n, j} \\
& =\sum_{j=1}^{s} c_{j} \sum_{n=1}^{\infty} \frac{[n+1+2 \beta(n+\alpha)] D_{n}(\lambda)}{2 \beta(1-\alpha)} a_{n, j}
\end{aligned}
$$

$\leq \sum_{j=1}^{\infty} c_{j}=1$. Therefore the proof is completed.

\section{Neighborhoods}

Now, we define (n, $\gamma$ )-neighborhood of a function $f \in \mathrm{S}$ by

$N_{\mathrm{n}, \gamma}=\left\{g \in \mathrm{S}: g(\mathrm{z})=z^{-1}+\sum_{n=1}^{\infty} b_{n} z^{n}\right.$ and

$$
\left.\sum_{n=1}^{\infty} n\left|a_{n}-b_{n}\right| \leq \gamma, 0 \leq \gamma<1\right\}
$$

For the identity function $e(\mathrm{z})=\mathrm{z}$, we have $N_{\mathrm{n}, \gamma}(\mathrm{e})=\left\{g \in \mathrm{S}: g(\mathrm{z})=z^{-1}+\sum_{n=1}^{\infty} b_{n} z^{n}\right.$ and $\left.\sum_{n=1}^{\infty} n\left|b_{n}\right| \leq \gamma\right\}$

Definition (9.1).A function $f \in \mathrm{S}$ issaid to be in the class $S^{\mu}(\lambda, \alpha, \beta)$ if there exists a function $g \in \mathrm{S}(\lambda, \alpha, \beta)$ such that

$$
\left|\frac{f(z)}{g(z)}-1\right|<1-\mu,(z \in U, 0 \leq \mu<1)
$$

Theorem (9.2) If $g \in \mathrm{S}(\lambda, \alpha, \beta)$ and

$\mu=1-\frac{\gamma[1+\beta(1+\alpha)](\lambda+1)(\lambda+2)}{[1+\beta(1+\alpha)](\lambda+1)(\lambda+2)-2 \beta(1-\alpha)}$

Then $N_{\mathrm{n}, \gamma}(g) \subset S^{\mu}(\lambda, \alpha, \beta)$.
Proof. Let $f \in N_{\mathrm{n}, r}(g)$,we want to find from (9.1) that

$$
\sum_{n=1}^{\infty} n\left|a_{n}-b_{n}\right| \leq \gamma
$$

Which readily implies the following coefficient in equality

$$
\sum_{n=1}^{\infty}\left|a_{n}-b_{n}\right| \leq \gamma,(n \in N)
$$

Since $g \in \mathrm{S}(\lambda, \alpha, \beta)$, hence we have from Theorem(2.1)

$$
\sum_{n=1}^{\infty} b_{n} \leq \frac{\beta(1-\alpha)}{[1+\beta(1+\alpha)](\lambda+1)(\lambda+2)}
$$

So that

$$
\begin{gathered}
\left|\frac{f(z)}{g(z)}-1\right|=\left|\frac{f(z)-g(z)}{g(z)}\right|=\left|\frac{\sum_{n=1}^{\infty}\left|a_{n}-b_{n}\right| z^{n+1}}{1+\sum_{n=1}^{\infty} b_{n} z^{n+1}}\right| \\
\quad \leq \frac{\sum_{n=1}^{\infty}\left|a_{n}-b_{n}\right|}{1-\sum_{n=1}^{\infty} b n} \leq \frac{\gamma}{1-\frac{\beta(1-\alpha)}{[1+\beta(1+\alpha)](\lambda+1)(\lambda+2)}} \\
\quad=\frac{\gamma[1+\beta(1+\alpha)](\lambda+1)(\lambda+2)}{[1+\beta(1+\alpha)](\lambda+1)(\lambda+2)-\beta(1-\alpha)}
\end{gathered}
$$

Then we get

$$
\left|\frac{f(z)}{g(z)}-1\right| \leq \frac{\gamma[1+\beta(1+\alpha)](\lambda+1)(\lambda+2)}{\left[\begin{array}{c}
1+\beta(1+\alpha)](\lambda+1)(\lambda+2)-\beta(1-\alpha) \\
=1-\mu
\end{array}\right.}
$$

Therefore, by definition(9.1), $f \in S^{\mu}(\lambda, \alpha, \beta)$

Thus by definition, $f \in S^{\mu}(\lambda, \alpha, \beta)$ for $\mu$ given by (9.2)

\section{Partial Sums}

Theorem(10.1). Let $f(z) \in \mathrm{S}(\lambda, \alpha, \beta)$ is given by (1.1) .Also $f_{1}(\mathrm{z})$ and $f_{k}(\mathrm{z})$ defined by

$$
\begin{aligned}
& f_{1}(\mathrm{z})=z^{-1} \quad \text { and } \\
& f_{k}(\mathrm{z})=\mathrm{z}^{-1}+\sum_{n=1}^{k} a_{n} z^{n},(\mathrm{n} \in \mathrm{N} /\{1\}) . \\
& \text { that } \\
& \sum_{n=1}^{\infty} d_{n}\left|a_{n}\right| \leq 1,
\end{aligned}
$$

Suppose that$$
\text { where }\left(d_{n}:=\frac{[n+1+2 \beta(n+\alpha)] D_{n}(\lambda)}{2 \beta(1-\alpha)}\right) \text {. }
$$

Furthermore,

$$
\operatorname{Re}\left\{\frac{f(z)}{f_{k}(z)}\right\}>1-\frac{1}{d_{k+1}}, \mathrm{z} \in U, n \in N
$$

And

$$
\operatorname{Re}\left\{\frac{f_{k}(z)}{f(z)}\right\}>\frac{d_{k+1}}{1+d_{k+1}} .
$$

Proof. For the coefficients $d_{n}$ given by (10.2)

$$
d_{n+1}>d_{n}>1 \text {. }
$$

Therefore,

$$
\sum_{n=1}^{k} a_{n}+d_{k+1} \sum_{n=k+1}^{\infty} a_{n} \leq \sum_{n=1}^{\infty} d_{n} a_{n} \leq 1
$$

By setting

$$
\begin{aligned}
g_{1}(\mathrm{z})=d_{k+1}\left\{\frac{f(z)}{f_{k}(z)}-\right. & \left.\left(1-\frac{1}{d_{k+1}}\right)\right\} \\
& =d_{k+1}\left\{\frac{f(z)}{f_{k}(z)}-1+\frac{1}{d_{k+1}}\right\} \\
= & 1+\frac{d_{k+1} \sum_{n=k+1}^{\infty} a_{n} z^{n+1}}{1+\sum_{n=1}^{k} a_{n} z^{n+1}}
\end{aligned}
$$

Applying (10.6), we get that

$$
\left|\frac{g_{1}(z)-1}{g_{1}(z)+1}\right| \leq \frac{d_{k+1} \sum_{n=k+1}^{\infty} a_{n}}{2-2 \sum_{n=1}^{k} a_{n}-d_{k+1} \sum_{n=k+1}^{\infty} a_{n}} \leq 1
$$

Which readily yields the assertion (10.3) of Theorem(10.1). If the function

\section{Volume 6 Issue 7, July 2017 www.ijsr.net}




\section{International Journal of Science and Research (IJSR) \\ ISSN (Online): 2319-7064}

Index Copernicus Value (2015): 78.96 | Impact Factor (2015): 6.391

$$
f(\mathrm{z})=z^{-1}+\frac{z^{k+1}}{d_{k+1}}
$$

$\frac{f(z)}{f_{k}(z)}=1-\frac{z^{k+1}}{d_{k+1}} \rightarrow 1-\frac{1}{d_{k+1}}$ as $\quad z \rightarrow 1^{-}$. This show the bound in (10.3).

Similarly, if we take

$$
\begin{aligned}
& g_{2}(\mathrm{z})=\left(1+d_{k+1}\right)\left\{\frac{f_{k}(z)}{f(z)}-\frac{d_{k+1}}{1+d_{k+1}}\right\} \\
& =1-\frac{\left(1+d_{k+1}\right) \sum_{n=k+1}^{\infty} a_{n} z^{n+1}}{1+\sum_{n=1}^{k} a_{n} z^{n+1}} \\
& \text { and making use of }(10.6), \text { we can deduce that } \\
& \quad\left|\frac{g_{2}(z)-1}{g_{2}(z)+1}\right| \leq \frac{\left(1+d_{k+1}\right) \sum_{n=k+1}^{\infty} a_{n}}{2-2 \sum_{n=1}^{k} a_{n}-\left(1+d_{k+1}\right) \sum_{n=k+1}^{\infty} a_{n}} \leq 1, \mathrm{z} \in U .
\end{aligned}
$$

Which leads to assertion (10.4) of Theorem (10.1).Therefore, the proof of Theorem(4.1) is complete.

Theorem(10.2). If $f(\mathrm{z})$ of the form (1.1) satisfies the Theorem (2.1). Then

$$
\operatorname{Re}\left\{\frac{f^{\prime}(z)}{f_{k}^{\prime}(z)}\right\} \geq 1-\frac{k+1}{d_{k+1}}
$$

Proof. By setting

$$
\begin{gathered}
g(\mathrm{z})=\frac{d_{k+1}}{k+1}\left\{\frac{f^{\prime}(z)}{f_{k}^{\prime}(z)}-\left(1-\frac{k+1}{d_{k+1}}\right)\right\} \\
=\frac{1+\frac{d_{k+1}}{k+1} \sum_{n=k+1}^{\infty} n a_{n} z^{n+1}+\sum_{n=1}^{k} n a_{n} z^{n+1}}{1+\sum_{n=1}^{k} n a_{n} z^{n+1}} \\
=1+\frac{\frac{d_{k+1}}{k+1} \sum_{n=k+1}^{\infty} n a_{n} z^{n+1}}{1+\sum_{n=1}^{k} n a_{n} z^{n+1}}, \text { Therefore, } \\
\left|\frac{g(z)-1}{g(z)+1}\right| \leq \frac{\frac{d_{k+1}}{k+1} \sum_{n=k+1}^{\infty} n a_{n}}{2-2 \sum_{n=1}^{k} n a_{n}-\frac{d_{k+1}}{k+1} \sum_{n=k+1}^{\infty} n a_{n}} \leq 1 .
\end{gathered}
$$

Now $\quad\left|\frac{g(z)-1}{g(z)+1}\right| \leq 1$ if

$$
\sum_{n=1}^{k} n\left|a_{n}\right|+\frac{d_{k+1}}{k+1} \sum_{n=k+1}^{\infty} n\left|a_{n}\right| \leq 1
$$

The result is sharp for the function

$$
f(z)=z+\frac{z^{k+1}}{d_{k+1}} .
$$

Theorem (10.3). If $f(\mathrm{z})$ of the form (1.1) satisfies the Theorem (2.1) then

$$
\operatorname{Re}\left\{\frac{f_{k}^{\prime}(z)}{f^{\prime}(z)}\right\} \geq \frac{d_{k+1}}{k+1+d_{k+1}} .
$$

Proof: By setting

$$
\begin{gathered}
g(\mathrm{z})=\frac{\left[(k+1)+d_{k+1}\right]}{\mathrm{k}+1}\left\{\frac{f_{k}^{\prime}(z)}{f^{\prime}(z)}-\frac{d_{k+1}}{\left[(k+1)+d_{k+1}\right]}\right\} \\
=1-\frac{\left(1+\frac{d_{k+1}}{k+1}\right) \sum_{n=k+1}^{\infty} n a_{n} z^{n+1}}{1+\sum_{n=1}^{\infty} n a_{n} z^{n+1}}
\end{gathered}
$$

and making use

$$
\sum_{n=1}^{k} n a_{n}+\left(1+\frac{d_{k+1}}{k+1}\right) \sum_{n=k+1}^{\infty} n a_{n} \leq 1 .
$$

We can deduce that

$$
\left|\frac{g(z)-1}{g(z)+1}\right| \leq
$$

$\frac{\left(1+\frac{d_{k+1}}{k+1}\right) \sum_{n=k+1}^{\infty} n a_{n}}{2-2 \sum_{n=1}^{k} n a_{n}-\left(1+\frac{d_{k+1}}{k+1}\right) \sum_{n=k+1}^{\infty} n a_{n}} \leq 1$.

Therefore, the result of Theorem (10.3) holds.

\section{References}

[1] W. G. Atshan, Subclass of meromorphic functions with positive coefficients defined by Ruscheweyh derivative
II, J. Surveys in Mathematics and its applications, 3(2008),67-77.

[2] W.G. Atshan and R.H. Buti, Some properties of a new subclass of meromorphic univalent functions with positive coefficients defined by Ruscheweyh derivative I , AL-Qad. J. Comp. Sci. \& Math., 1(2)(2009),36-43.

[3] B. A. Frasin and M. Darus, On certain meromorphic functions with positive coefficients, South east asian Bull. Math., 28(2004), 615-623.

[4] A. R. S. Juma and H. Zirar, On a class of Meromorphic univalent functions defined by hypergeometric function, Gen. Math. Notes, 1(2013), 63-73.

[5] L. Liu, A linear operator and its applications on meromorphic p-valent functions , Bull. Inst. Math. Acad. Sinica, 31(2003),23-32.

[6] L. Liu and M. Srivastava ,Classes of meromorphically multivalent function associated with the generalized hypergeometric functions , Math. Comput. Modell., 39(2004), 21-37.

[7] J. E. Miller, convex meromorphic mapping and related functions , proc. Amer. Math. Soc. , 25(1970) , 220-228

[8] M. L. Mogra , Hadamard product of certain meromorphic univalent functions J. Math. Anal.Appl.,157(1991),10-16

[9] R. K. Raina and H. M. Srivastava , A new class of meromorphically multivalent functions with applications of generalized hypergeometric functions , Math. Comput Modeling , 43(2006),350-356

[10]N. Xu and D. Yang , On starlikeness and close to convexity of certain meromorphic function, J. Korean Soc. Math. Edus. Ser B:Pure Appl. Math., [26] N. E. Cho and I. H. Kim, inclusion properties of certain classes of meromorphic functions associated with the generalized hypergeometric function ,Appl.Math. Comput., 187(2007), 115-121.

\section{Volume 6 Issue 7, July 2017 www.ijsr.net}

